\title{
Antibiotic resistance: turning evolutionary principles into clinical reality
}

Dan I. Andersson ${ }^{1}$, Nathalie Q. Balaban ${ }^{2}$, Fernando Baquero ${ }^{3}$, Patrice Courvalin ${ }^{4}$, Philippe Glaser ${ }^{5}$, Uri Gophna ${ }^{6}$, Roy Kishony ${ }^{7}$, Søren Molin ${ }^{8}$, and Tone Tønjum ${ }^{9,10}$

1 Department of Medical Biochemistry and Microbiology, University of Uppsala, Sweden

2. Racah Institute of Physics, the Hebrew University, Jerusalem, Israel

3. Department of Microbiology, Ramón y Cajal Health Research Institute, Madrid, Spain

4. French National Reference Center for Antibiotics, Institut Pasteur, Paris, France

5. Ecology and Evolution of Antibiotic Resistance, Institut Pasteur, Paris, France

6. School of Molecular Cell Biology and Biotechnology, Tel Aviv University, Tel Aviv, Israel

7. Faculty of Biology, The Technion, Haifa, Israel

8. Novo Nordisk Foundation Center for Biosustainability, Technical University of Denmark, Lyngby, Denmark

9. Department of Microbiology, University of Oslo, Oslo, Norway

10. Oslo University Hospital, Oslo, Norway

Correspondence to Uri Gophna (urigo@tauex.tau.ac.il); Roy Kishony (rkishony@technion.ac.il)

Conflicts of interests:

PC serves on the advisory board of Cequent Pharmaceuticals, Inc. that develops RNAi-based therapies.

\begin{abstract}
Antibiotic resistance is one of the major challenges facing modern medicine worldwide. The past few decades have witnessed rapid progress in our understanding of the multiple factors that affect the emergence and spread of antibiotic resistance at the population level and the level of the individual patient. However, the process of translating this progress into health policy and clinical practice has been slow. Here, we attempt to consolidate current knowledge about the evolution and ecology of antibiotic resistance into a roadmap for future research as well as clinical and environmental control of antibiotic resistance. At the population level, we examine emergence, transmission and dissemination of antibiotic resistance, and at the patient level, we examine adaptation involving bacterial physiology and host resilience. Finally, we describe new approaches and technologies for improving diagnosis and treatment and minimizing the spread of resistance.
\end{abstract}

Key words: Antibiotic resistance; evolution; transmission; rapid diagnostics; prevention; new therapy 


\section{INTRODUCTION}

The past few decades have witnessed rapid progress in our understanding of the many factors leading to the emergence and spread of antibiotic resistance, both at the population and individual patient levels. However, the process of translating this progress into health policy and clinical practice has been slow. While the message that antibiotics should be used responsibly in clinical practice and agriculture has been clearly stated and embraced in principle, and even adopted by policymakers, there has been little, if any, measurable change in clinical and agricultural practice, to reflect the urgency and magnitude of this public health crisis. With this in mind, the European Academy of Microbiology (EAM) organized a special working group, whose charge was to generate a roadmap to improve future research as well as clinical and environmental control of antibacterial /antimicrobial resistance (AMR), and prevent further escalation of this public health crisis.

\section{Population level analysis I}

\section{Emergence and transmission of antibiotic resistance}

With regard to the emergence, transmission and dissemination of antibiotic resistance, several unresolved issues need to be addressed in the immediate future. The most important of these issues are: (i) In what context or biological niche do resistant bacteria, antibiotic resistance genes (ARGs) and mobile genetic elements (MGEs) that carry ARGs arise and emerge? Does resistance emerge primarily in humans or animals during treatment, or in bacteria that exist outside of a host organism, in an environmental niche? Emergence in this context encompasses both the appearance and the dissemination of the resistant lineage or MGE carrying ARGs. (ii) What selective pressures drive the evolution of resistance (i.e. antibiotics, biocides, heavy metals or pharmaceuticals other than antibiotics)? (iii) Which bacterial characteristics are critical determinants of the rate of resistance evolution (i.e. mutation rate, fitness cost of resistance and of MGE carriage, pharmacodynamic response curves combined with drug pharmacokinetics etc.)? (iv) What specific properties contribute to global dissemination of antibacterial drug- or multi-drug-resistant bacteria? and ( $v$ ) What are the roles of host-associated factors, such as population density, host immunity and vaccination, travel, hygiene, and healthcare systems on the emergence and transmission of resistance? To address these questions, global epidemiological and genomic data as well as microbiome data will be needed, as well as diverse laboratory studies of antibiotic resistant bacteria. The latter should encompass experimental evolution, the study of resistant phenotypes, mutation mapping, persistence, genetic mobility and more. Those research questions must be addressed in detail, and rational evidence-based strategies must be implemented to reduce the rates of emergence dissemination of resistance. A concerted effort will also be needed to identify novel antibiotic drugs that can be expected to remain highly-effective bacteriocides well into the future.

\section{Emergence of antibacterial resistance}

(i) Where do resistant bacteria emerge? A resistant bacterium could exist in any environment, but the frequency of the resistant genotype/phenotype in the population is likely to increase when it confers increased survival capacity under selective pressure (Fig. 1). While there are 
many types of selective pressures, for the purpose of the current discussion, we will assume that the evolution of antibiotic resistance is driven primarily by the exposure to antibiotic drugs (van de Sande-Bruinsma, et al., 2008). Nevertheless, indirect factors such as GDP (Growth Domestic Product) per capita, public healthcare spending, an aging population, increase in travel or poor governance can be strongly correlated with resistance (Collignon, et al., 2018).

It is often assumed that resistance emerges when the parental drug-sensitive bacteria are exposed to antibiotics in an infected animal/human host organism; for example, when an infection is subjected to long-term antibiotic drug therapy. In such cases, resistance usually emerges by mutation, rather than by Horizontal Gene Transfer (HGT). For example, cystic fibrosis patients carry abundant populations of Pseudomonas aeruginosa that are subject to strong druginduced selective pressure over a long time. Population turnover in such patients is relatively low, allowing resistant bacteria to emerge and rapidly increase in number (Oliver, et al., 2000). A contrasting scenario involving HGT is illustrated by the emergence of vancomycin-resistant Staphylococcus aureus, where high-level vancomycin resistance encoded by the vanA gene cluster on the transposon Tn 1546 was acquired from co-infecting enterococci (Weigel, et al., 2003, Courvalin, 2006). This example highlights an important aspect of the role of HGT in resistance: while HGT might often lead to the emergence of resistance, it is not sufficient to create a resistance problem in the clinical sense. Indeed, the vanA gene in $S$. aureus has spread poorly and has not become a major health problem worldwide. Success in transmission is thus as important (or even more important) than emergence, and is generally facilitated by antibiotic selection. Another example is acquisition of resistance to linezolid used to decolonize patients before surgical intervention through HGT involving episomal plasmids carrying cfr (the chloramphenicol-florfenicol resistance gene), leading to high resistance to linezolid (Dortet, et al., 2018). A third scenario is when a pathogenic bacterium is already resistant when it infects an individual patient, and the time and place of acquisition of resistance remain unknown. Resistance could have emerged in a previously antibiotic-treated patient or in another niche (e.g. animals, wastewater treatment facility, manure tanks, hospital-environment). This perspective is emphasized in the "One-Health" approach (Hernando-Amado, et al., 2019).

An example in which antibiotic use in animals is likely to have caused a resistance problem in humans is plasmid-borne colistin resistance due to the $\mathrm{mcr}-1$ (mobilized colistin resistance) gene. This gene has been detected in clinical isolates of Escherichia coli, Salmonella enterica, Klebsiella pneumoniae and Enterobacter aerogenes/cloacae, and several lines of evidence indicate that it was the extensive use of colistin as a growth promoter in livestock that has enriched for it and has facilitated its subsequent transmission to humans. Colistin has been widely used in animals for many decades, while use in humans is rare, due to toxicity, and mcr-1 is detected much more frequently in animal isolates than in human isolates (Liu, et al., 2016, Schwarz \& Johnson, 2016). Overall, these findings indicate that bacteria carrying $m c r-1$ were selected for in animals that received colistin and those bacteria or plasmids carrying $\mathrm{mcr}$ genes were later transmitted to humans where they became a clinical concern. Other examples of such animal to human (or viceversa) transmission have been observed for MRSA [methicillin-resistant Staphylococcus aureus (Price, et al., 2012, Frana, et al., 2013, Rinsky, et al., 2013)]. This raises the question: Is the spread of resistance from animals to humans exceptional or typical? At present, it is difficult to answer this question with any degree of certainty. However, based on limited data, animal use is likely 
to contribute significantly to AMR for some antibiotics and bacterial pathogens, particularly in environments allowing the coalescence of human and animal microbiomes (Hernando-Amado, et al., 2019).

Host-specificity can be a strong limiting factor, and transmission of resistant bacteria between animals and humans is complex, generally requiring specific bacterial adaptations to the human and animal hosts. Yet, when resistance involves mobile genes and plasmids, there is also strong potential for direct genetic transmission through mechanisms involving DNA recombination, as observed for plasmid-borne mcr-1 (Wang, et al., 2018). Indeed, a systematic survey of E. coli from human and animal origins revealed very few potential cases of animal-to-human transmission of resistance through expression of Extended-Spectrum Beta-Lactamases (ESBL) (Ludden, et al., 2019). Future studies are required to further characterize the evolutionary dynamics of MGEs, co-adaptation between resistance-vectors (plasmids, integrative and conjugative elements, transposons etc.), ARGs and bacteria, in order to correctly identify the source of antibiotic resistance (human, animal or environmental) and to limit its spread. Notably, that transmission of antibiotic resistance to relevant human pathogens probably starts in phylogenetically-related species able to exploit animal and environmental niches. Such "generalist" lineages that can integrate into the microbiota of both humans and animals are of particular importance for the One-Health prevention of spread of antibiotic resistance. The transmission of these resistant species or clones is certainly facilitated by the "globalization of microbiotas", resulting from the reduction in the diversity of food animals and by the decrease in gut microbial diversity by "westernization processes" (Segata, 2015).

In the environmental niche, a number of factors are known to exert strong selective pressure for acquiring resistance to antibiotics [Figure 1., (Yoon, et al., 2016, Yoon, et al., 2017)]. These include natural production of antibiotics by microbes, influx of human-generated waste enriched for excreted antibiotics (depending on the antibiotic class, up to $80 \%$ of an antibiotic administered to humans is excreted via urine in an unchanged chemical form, the most stable classes being glycopeptides, aminoglycosides and fluoroquinolones), influx of antibiotics used in animal production and aquaculture, and pollution from pharmaceutical manufacturing. Indeed, a substantial fraction of the chemically stable forms of the antibiotics used in animal husbandry, chemical production, aquaculture and human therapy ultimately end up in the external environment (rivers, lakes and soils) and/or in food products, where they can potentially continue to exert their effect. Although the selective pressures in such environments (with the exception of antibiotic production plants) tend to be weak, recent results show that even very low antibiotic concentrations can have substantial selective effects (Gullberg, et al., 2011, Liu, et al., 2011, Gullberg, et al., 2014, Wistrand-Yuen, et al., 2018). However, it is difficult to determine whether an increased abundance of antibiotic resistance genes in water or soil reflects the presence of residual antibiotics in that environment (Lundstrom, et al., 2016), or rather contaminating bacteria from human or animals excrements (Karkman, et al., 2019). It is noteworthy that selection by environmental antibiotics released by the pharmaceutical industry may favor taxonomic shifts towards intrinsically resistant species or strains harboring chromosomal resistance mutations rather than acquisition of mobile resistance determinants harboring resistance genes (Bengtsson-Palme, et al., 2019). 
(ii) Which selective pressures drive the evolution of antibiotic resistance? It is probably safe to say that exposure to antibiotics is the main driver, considering biological first principles and the fact that higher volume of antibiotic use correlates relatively well with frequency of resistance (van de Sande-Bruinsma, et al., 2008). However, that does not exclude possible roles for additional factors, such as heavy metals and other biocides, either by co-selection between biocide and antibiotic resistance genes (i.e. where a multi-resistance gene element (e.g. MGE) provides resistance to both, (Gullberg, et al., 2014, Pal, et al., 2015, Wales \& Davies, 2015, Flach, et al., 2017) or via cross-resistance between the biocide and antibiotic resistance mechanisms (Wales \& Davies, 2015, Webber, et al., 2015, Maillard, 2018). Biocides could also reduce the colonization resistance (the barrier effect) of the naturally occurring microbiota of that environment and thus facilitate its invasion by antibiotic resistant bacteria, but such effects have not yet been adequately studied. High levels of primarily non-antibiotic pharmaceuticals are released into the environment, and can influence the microbiota including psychotropics, statins, or analgesic and anti-inflammatory compounds, which might produce shifts in the microbial population structure, eventually favoring antibiotic-resistant bacteria (Boxall, 2004, Gatica \& Cytryn, 2013, Ko, et al., 2017, Zimmermann \& Curtis, 2018, Cussotto, et al., 2019). Our understanding of such influences is limited by the lack of sufficient quantitative data on relative levels of antibiotics and resistance genes in environments other than wastewater treatment plants and hospitals.

(iii) Which bacterial factors are critical determinants of the rate at which antibiotic resistance evolves? Irrespective of whether the resistance is acquired via vertical (mutation) or HGT, its evolution, transmission, and maintenance in a population of bacteria is driven by a complex interplay of several factors (Hughes \& Andersson, 2017). Primary factors include the mutation supply rate [i.e. the rate at which resistance genes/mutations arise which is determined by population sizes $x$ rates of mutation and horizontal gene transfer (Andersson, 2015, Martinez, et al., 2015, Sommer, et al., 2017)], the level of resistance conferred by the resistance mechanism, the growth of the resistant mutant at different drug concentrations [the pharmacodynamic response curve (Yu, et al., 2018)], and the strength of various selective pressures (Andersson \& Hughes, 2010, Gullberg, et al., 2011, Oz, et al., 2014, Huseby, et al., 2017, Wistrand-Yuen, et al., 2018). The level of neutral allelic variation in the population can also affect the probability of mutation to a resistant genotype, because neutral nucleotide substitutions can reduce the number of steps required to produce an antibiotic-resistant target protein.

It is also notable in view of this complexity, that current preclinical assessments of the risk of resistance development for new antibiotics drug candidates often focus exclusively on de novo mutational resistance, typically using mutation rate determinations during serial passage procedures under defined laboratory conditions. This narrow focus is highly limited if not downright inappropriate for two reasons. First, in vitro mutation rates do not correlate with the clinical rate of emergence of resistance. The primary reason for this discrepancy is probably that resistance evolution and fixation of a resistant mutant within a patient are not strongly limited by the bacterial mutation supply rate (Andersson, 2015). Second, in the majority of human pathogens the resistance mechanisms involve the horizontal acquisition of pre-existing resistance genes, and HGT frequency is difficult to assess using in vitro experiments. 
Secondary factors include compensatory mutations at other sites that reduce the fitness cost associated with resistance (Bjorkman, et al., 2000, Marcusson, et al., 2009, Andersson \& Hughes, 2010, San Millan, et al., 2014), and epistatic interactions between different resistance mechanisms that influence both the level of resistance and overall fitness (Trindade, et al., 2009, Silva, et al., 2011, de Visser \& Krug, 2014, Knopp \& Andersson, 2015, Moura de Sousa, et al., 2017, Durão, et al., 2018, Knopp \& Andersson, 2018). Furthermore, cross-resistance, collateral sensitivity (Lazar, et al., 2014, Munck, et al., 2014, Oz, et al., 2014, Roemhild, et al., 2018, Rosenkilde, et al., 2019), and co-selection, where one resistance gene hitchhikes with another gene because of their genetic linkage (Weinroth, et al., 2018), can have strong effects on both the rate and trajectory of resistance evolution. Resistance to multiple antibiotics is acquired through a complex adaptation process that progresses via sequential independent acquisition of ARGs and/or mutations that may occur in different niches (human, animal, external environment). Furthermore, recent studies suggest that antibiotic resistance may depend on environmental conditions and multispecies bacterial interactions in microbial communities, such as the presence of microbial predators (Cairns, et al., 2018, Hiltunen, et al., 2018), or of specific growth conditions (Kubicek-Sutherland, et al., 2015) or metabolites (Thulin, et al., 2017) that can modulate and alter the phenotypic expression of a specific resistance mechanism (Hughes \& Andersson, 2017). For example, competitive interactions among bacterial organisms, may act as an indirect selection pressure for antibiotic resistance; e.g. highly antibiotic-resistant, transmissible and pathogenic $E$. coli strains that are frequently producers of microcins, suppressing closely related susceptibile bacteria (Micenkova, et al., 2016).

In summary, bacteria are exposed to a number of different selective agents in complex mixtures and diverse environments that have time- and space-variable metabolite concentrations, as well as bacterial competitors, phages and other viruses, fungi, proteobacterial predators and protozoa. These variables create a complex web of possibilities and selection pressures, and it is very hard to disentangle the relative contributions and importance of different factors, or to identify which factors can be manipulated to our advantage.

\section{Dissemination and transmission of antibiotic resistance}

(i) What determines the global dissemination of specific clones? Molecular and genomic epidemiology provide evidence that nearly identical MDR strains can be isolated independently and at geographically distant locations. This suggests that a few specific lineages, which appear to be especially successful contribute disproportionately to the antibiotic resistance burden worldwide. For example, the global dissemination of the gene encoding ESBL enzyme CTX-M15 correlates with the global dissemination of a specific lineage of E. coli known as ST131 H30-RX. The emergence of this $E$. coli lineage at the beginning of the $21^{\text {st }}$ century has been extensively studied, and associated with the progressive acquisition of fluoroquinolone and $\beta$-lactam resistance (Price, et al., 2013). However, the very emergence of this lineage, might have preceded the acquisition of antibiotic resistance and the reasons for its success remains elusive (Kallonen, et al., 2017, Yair \& Gophna, 2018). Similarly, K. pneumoniae clonal group 258 that expresses the carbapenemase KPC and is considered to be responsible for most infections by hospital-acquired carbapenem-resistant K. pneumoniae (Wyres \& Holt, 2016). However, again, no specific properties have been identified that might explain the "hospital-adaptation" of this clone, and this lineage is common in the US, Italy, Greece and Israel, but rare in France, Germany and the 
UK. In southeast Asia, a related lineage, ST11, seems to dominate. This situation may be common to a broad range of opportunistic pathogens as it was also observed among community acquired MRSA with the dominance of the USA300 lineage in the US (Planet, et al., 2013) and of the clonal complex 80 in Europe and in North Africa (Stegger, et al., 2014). In Streptococcus agalactiae, a few tetracycline resistant lineages replaced preexisting strains worldwide after 1950, when tetracycline use increased (Da Cunha, et al., 2014). The global spread of these lineages is associated with the emergence of neonatal group B streptococci (GBS) infections in Europe and the US in the 1960s. However, aside from the past use of tetracycline the reasons for the emergence of these lineages remain unknown. Despite rare current clinical use of tetracycline in humans (while commonly used as growth promoters for livestock in the USA), the rate of resistance to this drug remains close to $100 \%$ in Western countries. This illustrates how little we understand the global dissemination of specific bacterial lineages that contribute disproportionately to the global emergence of antibiotic resistance.

(ii) What are the roles of human societal factors such as population density, host immunity and vaccination, travel, hygiene, and human healthcare systems in the emergence and transmission of antibiotic-resistant bacteria? While societal factors fall outside the scope of this statement paper, they nevertheless have a high impact (Collignon, et al., 2018) and are crucial for controlling the spread of resistance worldwide. In the One-Health scope, increase in population density of humans, food animals, and plants, associated with westernized dietary habits, particularly the increase in animal production and meat consumption in low and middle income countries, and the green revolution in agriculture, provides a landscape that shapes the current dimensions of antibiotic resistance (Van Boeckel, et al., 2019, Baquero, et al., In press).

(iii) What role do complex microbiomes play in emergence and spread of antibiotic resistance? For many infections, including most urinary tract and blood infections, the pathogen is a single entity, often one clone that then encounters the drug. At the site of infection, this pathogen is in genetic isolation, thus, unable to survive exposure to the drug except by intrinsic mechanisms of resistance or tolerance, or mutational variation. However, the invasion of tissue is frequently preceded by colonization of mucosal surfaces of the gastrointestinal tract, the upper respiratory tract, or the skin, where the pathogen may acquire antibiotic resistance through HGT. The history of antibiotic exposure of the host (patient) determines the density of potential donors. During antibiotic therapy, the microbiomes of such body sites are disturbed, and bacterial diversity is thereby decreased, while the microbiome can serve as a reservoir for AMR-related genes and signatures. Although the bacterial community bounces back after treatment, it may not fully return to its pre-treatment state (Dethlefsen \& Relman, 2011). Of course, antibiotic treatment can also provide a minor member of the microbiome that is at least partially resistant to the drug with a chance to expand at the expense of susceptible bacteria, some of which may actually go extinct following treatment. A number of critical gaps in our understanding of antibiotic resistance in microbiomes remain. These include indirect resistance and the effect of within community HGT. However, metagenomic observations should be supported by additional experimental evidence to conclusively establish causation or mechanism.

Indirect resistance. Indirect resistance is the cross-protection that antibiotic resistant strains that detoxify antimicrobial drugs can provide to antibiotic-susceptible members of the 
microbiome, a phenomenon that has been demonstrated in vitro (Baquero, et al., 1985, Nicoloff \& Andersson, 2016, Yurtsev, et al., 2016). However, how indirect resistance operates in the context of an in vivo microbial community is unknown, and early investigations have already unraveled some surprising results. For example, antibiotic-resistant strains can in some circumstances be outcompeted by antibiotic-susceptible ones in the presence of an antibiotic (Sorg, et al., 2016).

Barriers to HGT and transferable resistance. The microbiomes of body sites have been shown to have higher rates of HGT than other ecosystems (Smillie, et al., 2011), which should result in higher rates of resistance (see above), but despite the fact that many new genes in the microbiome confer antibiotic resistance to $E$. coli when expressed under their own native promoter none of those genes has ever been identified in E. coli or any other pathogen (Sommer, et al., 2009). Since antibiotic resistance genes tend to be over-represented on MGEs, and were transmitted from soil bacteria to human pathogens (Forsberg, et al., 2012), there must be forces that restrict the horizontal transmission of resistance, yet we know little about those barriers. $A$ recent study showed for metallo- $\beta$-lactamases that interactions between each acquired gene and multiple cellular processes of the bacterial host (transcription, translation, and translocation) could influence the genetic incompatibility and efficiency of HGT (Socha, et al., 2019).

Fortunately, the scientific infrastructure and technology required to address these knowledge gaps already exists. Metagenomics is one of the preferred approaches and has become affordable. The metagenomics toolbox has matured and can be successfully applied to investigate taxonomy (Segata, et al., 2012), microbial pathways (Abubucker, et al., 2012) and antibiotic resistance (Lakin, et al., 2017, Lanza, et al., 2018, Ruppe, et al., 2019). The challenge will be to use metagenomics in clinical studies involving human patients that can ideally be followed up before, during and after different antibiotic interventions. In this manner, so that microbial community factors that affect resistance could potentially be addressed in a meaningful manner. Convincing clinical metagenomics studies have been carried out in a small number of healthy volunteers (Suez, et al., 2018). The next logical step is to expand such methodology to clinical trials in larger patient cohorts. The objectives are: 1) to document the effect of antibiotics on the normal microbiota; as the collapse in diversity facilitates the growth of antibiotic resistant bacteria, 2) to document changes in the intestinal resistome, detecting the expansion of "high-risk" antibiotic resistance genes. While challenging, especially in terms of data management and analysis, this next step is essential given the high inter-individual variation in human microbiome composition.

\section{Population level analysis II}

Future projections: Strategies to mitigate or prevent antibiotic resistance

(i) Reduce selective pressure. The most direct and efficient way to reduce the emergence of antibiotic-resistance microbes is to reduce the relevant selective pressures that provide them with a fitness advantage. It is therefore critical to identify the selective factors whose reduction will be most effective in limiting emergence of resistance. Hospitals are hubs of transmission of antibiotic resistance because of the high local use of antibiotics and the high density of infectionsusceptible hosts. However, antibiotic stewardship, hospital hygiene and optimized (e.g. more cautious) use of antibiotic drugs in the hospital setting are obvious steps to reduce the selective pressure for antibiotic resistance (Baur, et al., 2017). Nevertheless, given that approximately 
$90 \%$ of antibiotic use in humans occurs outside hospitals and the risk for dissemination of MDR clones in the community, it would seem also reasonable to focus more heavily on community use, especially for the most common infections, e.g. respiratory and urinary tract infections (Low, et al., 2018). This would require rapid tests to distinguish viral from bacterial respiratory infections and antibacterial susceptibility tests (ASTs), so that choice of antibiotic drugs is evidence-based rather than empirical, and that treatment can deescalate more rapidly. A related question, for which we at present do not have a good answer, is to what extent antibiotic use in animals (therapeutically and for growth promotion in certain countries) contributes to the emergence of resistance in human-relevant pathogens and consequently what effect a reduction in antibiotic use in animals will have on resistance in humans. Data from the Netherlands, a country that had reduced antimicrobial use in poultry and pigs for more than a decade, have been encouraging, showing for example a significant reduction in antibiotic-resistant $E$. coli in the pig and veal calf production sectors (Dorado-García, et al., 2016). Although the contribution of exposure to pig and veal products to ESBL-producing E. coli is only modest (Mughini-Gras, et al., 2019), it would nevertheless be wise to be as restrictive as possible, and globally ban the use of antibiotics to promote growth in livestock, as has been done in EU since January 1, 2006. Regarding the use of biocides and metals such as silver in various contexts, their role in driving antibiotic resistance is uncertain but based on available laboratory experiments, they should also be used with caution (Wales \& Davies, 2015, Webber, et al., 2015, Maillard, 2018).

(ii) Develop antibiotics against novel targets, with low propensity for resistance. An interesting idea is whether it is possible to identify therapeutic targets that are less prone to suffer loss of efficacy over time, due to emergence of resistance and focus drug development on these targets. To do this, the most important bacterial factors for sustained drug efficacy must be identified (see section (iii) above). For mutational resistance, the ideal target will have a high fitness cost when mutated, and a slow and inefficient rate of compensatory evolution, while the ideal drug should have a steep pharmacodynamic response in both susceptible and resistant bacteria. The selective window should be as narrow as possible, such that the time spent during treatment within the selective window is minimal. Notably, in vitro mutation rates have to date been poor predictors of the rate of emergence of clinical resistance, for antibiotics in current use (Andersson, 2015, Sommer, et al., 2017).

Newly developed antimicrobials that exhibit no cross-resistance with previous drugs should act equally well on microbes that are susceptible or resistant to older antibiotics. In this scenario, if the proportion of "old-drug"-resistant strains is low, those populations can be driven extinct, while "old-drug"-susceptible bacteria will have higher chances of survival due to their higher density and prevalence. In fact, in the 1980's the introduction of several new antibiotics was associated with decrease of resistance to older ones (Gerding, et al., 1991). If on the other hand, the proportion of resistant organisms currently exceeds that of the susceptible, the introduction of a new drug might actually eliminate the susceptible minority population and enrich for the resistant organisms.

The considerations regarding HGT-mediated resistance, are quite different than those for mutational resistance. Relevant parameters for minimizing HGT include: (i) whether potential genes that confer resistance to the chosen molecule or related compounds, exist naturally or can evolve to provide resistance with few mutations, (ii) the ecological opportunity of HGT, (iii) the rate of HGT, (iv) the fitness effects on the recipient bacteria after HGT (Sommer, et al., 2017) and 
(v) the likelihood that resistance genes move between various HGT vectors with different propensity for HGT. It is interesting to note that even though all of these parameters can today be experimentally determined, they are rarely investigated and used by academia/industry as a basis for a rational risk assessment.

(iii) Reduce mutation supply and HGT. Two approaches have been used to date to reduce the supply of mutations: first, drugs that inhibit processes or functions (e.g. LexA, Mfd) that increase mutation rates (Cirz, et al., 2005, Smith \& Romesberg, 2007, Ragheb, et al., 2019) , and second drugs that inhibit HGT via conjugative plasmids (Getino \& de la Cruz, 2018). Both of these approaches are very interesting but they suffer from the assumption that the mutation supply rate is rate-limiting for resistance evolution in natural settings (see section above). Furthermore, the development of such drugs is at least as difficult as that of new bona fide antibiotics and the indications for their use are undetermined. It is also unclear how one would test the clinical efficacy of such drugs in phase 2-3 trials. Presumably, the anti-mutagenesis drugs would be given in combination with antibiotics in treated patients where resistance is known to evolve through mutation (e.g. M. tuberculosis). For conjugation-inhibitors, since the emergence of the resistant bacteria via HGT often occurs outside of treated patients, those drugs may be better suited to testing and treatment in high transfer risk environments, for example in animal husbandry, rather than in human patients.

(iv) Narrow the window of selection. Frequently, resistant organisms have an antibiotic concentration-dependent selective advantage only in a specific segment of the gradient of antibiotic concentrations (concentration-dependent selection), as very low concentrations are devoid of any effect, while very high ones might completely inhibit the resistant cells (Baquero \& Negri, 1997). This segment is the "window of selection" (Drlica \& Zhao, 2007), and the longer these selective antibiotic concentrations prevail during the infection or at the colonization site, the larger the risk that resistance will emerge. Furthermore, selective concentrations for resistant variants could be extremely low, and still non-lethal susceptible organisms in the population (Hughes \& Andersson, 2012). When antibiotics with longer half-life are used the risk of selecting for resistant organisms is higher (Baquero \& Negri, 1997). For optimized antibiotic therapy, pharmacokinetic/pharmacodynamic (PK/PD) modeling and simulation should underpin rational antibiotic therapy to secure efficacy and safety in clinical practice. The steepness of the antibiotic gradient over time are functions of the PK/PD properties of each antibiotic. The application of PK/PD model-based dose individualization to optimize therapy is about to become a core feature of precision medicine. These PK/PD characteristics, along with different drug and dosing regimens are tools that can be used to narrow the window of selection and minimize the risk that antibiotic-resistant organisms will emerge.

(v) Exploit collateral sensitivity. Collateral sensitivity (CS, also known as the seesaw effect) occurs when evolution of resistance to one drug increases susceptibility to another drug or drug class. CS has emerged as an interesting new idea that could potentially be used to reduce the rate of emergence of resistance in clinical settings. CS has probably already prevented the spread of some types of antibiotic resistance (Perichon \& Courvalin, 2009). Under laboratory conditions, $\mathrm{CS}$ can be relatively common and can reduce the rate at which resistance emerges (Imamovic \& 
Sommer, 2013, Lazar, et al., 2013, Kim, et al., 2014, Munck, et al., 2014, Gonzales, et al., 2015, Lazar, et al., 2018, Podnecky, et al., 2018, Nichol, et al., 2019, Rosenkilde, et al., 2019). CS is related to the phenomenon of cellular hysteresis, which is the long-lasting change in cellular physiology that is induced by one antibiotic and sensitizes the bacteria to another subsequently administered antibiotic (Roemhild, et al., 2018). Whether these evolutionary-informed approaches will provide useful clinical benefits remain unclear and it will depend on numerous factors, including the identification of clinically relevant drug-pairs, the magnitude of the CS (which in most cases is small), whether the same trajectories observed in in vitro evolution experiments apply to clinical settings (Podnecky, et al., 2018, Nichol, et al., 2019), and whether $\mathrm{CS}$ is a conserved phenomenon among different species and clinical isolates. Such questions can and should be resolved experimentally and they have already inspired interest in combined sequential use of antibiotics. Generally, the earlier the switch to the second-line drugs, the better the effect in terms of restricting resistance (Haber, et al., 2010). Another related idea is spatial "mixing", which is based on ecological theory. This approach postulates that a combination of drugs in space, e.g., where patients in a given hospital ward are treated with either antibiotic $A$ and others with antibiotic $B$, will reduce the chance of emergence of resistant organisms (Bergstrom, et al., 2004).

(vi) Improve healthcare practice and implement evidence-based health policy relevant to reducing antibiotic resistance. Overuse of antibiotic drugs will ultimately weaken their efficacy against human pathogens, while more judicious use of antibiotics will help preserve the efficacy of antibiotics against infectious disease, protecting public health, with great benefits at both the societal and the individual levels. An individual's personal history of antibiotic exposure is probably correlated with the likelihood of carriage of resistant opportunistic pathogens, increasing the risks of therapy failure under future conditions of greater frailty (Baquero, 2007). In many cases, antibiotics are prescribed to treat mild, self-limiting community-based infections, possibly without adequate awareness that such use contributes to global antibiotic pollution and resistance. In such mild infections, it is important that use of non-antibiotic therapy, such as antiinflammatory drugs [e.g. ibuprofen, (Baquero, et al., 2015) should be considered, while using biomarkers, such as procalcitonin and lactate, to monitor patients for bacterial infection and sepsis (Branche, et al., 2019). Moreover, for mild infections of immunocompetent hosts bacteriostatic antibiotics are as useful as bactericidal ones (Nemeth, et al., 2015), and short therapeutic courses are generally sufficient.

Another important healthcare practice is population-wide vaccination against viruses, such as influenza, which reduces host susceptibility to bacterial infections (Kwong, et al., 2009, Kash \& Taubenberger, 2015). The rationale is based on three factors: first, it minimizes the number of cases where diagnostic uncertainty favors antibiotic usage; second, it reduces the number of antibiotic-treatable co-infections that are secondary to viral illnesses; and third, it prevents the increased transmission rate of resistant bacterial pathogens between hosts that is associated with viral infections. Similarly, if the length of hospital-stays are kept as short as possible, the turnover and absolute number of patients admitted to the hospital can increase; this can ultimately reduce the local prevalence of antibiotic-resistant organisms (Lipsitch, et al., 2000). Antibiotic-resistant bacteria (e. g. following hospitalization in a high risk area for transmission) can be detected using rapid diagnostic tools before a new patient is admitted to the hospital in 
areas at high risk for transmission, and containment measures for patients that are colonized with antibiotic-resistant organisms can be implemented as quickly as possible.

(vii) Antibiotic combinations. During the last quarter of the 20th century, a global campaign was mounted against use of antibiotic combinations. At present, it may be time for better-informed and different healthcare practice regarding combination therapy. Indeed, for most infections, combination therapy is not much more effective than monotherapy with a single drug. However, combination therapy reduces the risk that resistant organisms will emerge, as variants that are resistant to drug $A$ remain susceptible to drug $B$, and vice versa. This is clear in specific infections; the typical case-in-point being tuberculosis, but is also relevant for infections in immunosuppressed patients. In fact, antibiotic combinations should be considered a strategy to extend the life of antibiotics (Tyers \& Wright, 2019) and reduce the emergence of antibiotic resistance (Mebis, et al., 1998). Evolution-informed use of antibiotic combinations, based on a thorough understanding of the spectrum of resistance to individual drugs, should enable the identification of the combinations that are not only efficacious but also less prone to resistance. Moreover, In a number of cases, single-antibiotics are ineffective in eradicating otherwise apparently susceptible pathogens because these pathogens are only partially-susceptible to them under in vivo conditions, frequently because of slow growth, heteroresistance or persistence (see below), sometimes known as a dormant stage. In these cases (endocarditis, endovascular infections, cystic fibrosis pathogenic colonization), specific antibiotics (such as aminoglycosides) targeting these dormant populations are frequently added, generating a combination therapy that is more effective than single drugs alone. Of note, antibiotic combinations that include drugs whose resistances are associated with a single bacterial species might actually favor multi-resistance due to co-resistance. Thus, combinations should be selected carefully according to the specific clinical case. A combination therapy that has been in clinical use since the 1960s is trimethoprim-sulfamethoxazole that synergistically attack two different targets in the pathway of tetrahydrofolic acid synthesis (Masters, et al., 2003). Of course, concomitant use of two antibiotic molecules does not guarantee that both reach the same bacterial cell, and there is always a certain degree of in vivo de-combination. This limitation could possibly be overcome by using a nanocarrier as delivery vehicle for both drugs (Walvekar, et al., 2019).

(viii) Antibacterial vaccination. Antibacterial vaccination can be a highly effective intervention against antibiotic-resistant human bacterial pathogens (Jansen, et al., 2018). This has been unequivocally demonstrated in the case of antibiotic-resistant Streptococcus pneumoniae: the polysaccharide-protein conjugate vaccines PCV7 and PCV13 have been used successfully, targeting the most frequent serotypes that are resistant to $\beta$-lactams, macrolides and fluoroquinolones. Similarly, anti-Haemophilus influenzae type B (Hib) vaccination significantly reduced $\beta$-lactamase-producing isolates. For $E$. coli, a promising bioconjugate vaccine that contains the O-antigens of four E. coli serotypes (ExPEC4V (Huttner \& Gambillara, 2018)) has been developed. Moreover, efficacious oral vaccines have been efficacious against Salmonella (Kantele, et al., 2012, Wahid, et al., 2015, Wahid, et al., 2016) and similar vaccines may be developed in the future to reduce gut colonization by resistant Enterobacterales. Candidate vaccines against $S$. aureus are being evaluated, in particular the 4-antigen vaccine (SA4Ag, 
(Begier, et al., 2017)) that might influence resistance frequencies in this organism. Note that we can envisage two main scenarios for vaccination: 1) massive vaccination; and 2) groupvaccination (for instance healthcare workers or in specific hospital wards at risk for high incidence of resistance). A phenomenon known as "herd immunity" extends the protection to non-treated individuals as follows: as the proportion of carriers of resistant organisms decreases in the vaccinated individuals, the rate of new colonization by resistant organisms decreases in the nonvaccinated ones as well.

Passive vaccination can also be used to combat resistance. Antibiotic resistance frequently emerges in immunocompromized patients (DeNegre, et al., 2019), including newborns, the elderly, patients undergoing chemotherapy or organ transplants, and in specific biological contexts where immune-surveillance is absent (catheters, biofilms). This suggests that immunity plays a direct role in preventing or delaying the emergence of antibiotic-resistant organisms. Phagocytosis reduces the bacterial load and therefore can directly eliminate bacterial variants with mutational or acquired resistance (Levin, et al., 2017), while antibodies act synergistically with antibiotics in bacterial elimination. The use of polyclonal or monoclonal antibodies against high-risk resistant bacterial clones or against specific mechanisms of resistance, in combination with antibiotics, might therefore be a useful future strategy (Saylor, et al., 2009, Al-Hamad, et al., 2011). By using e.g. cutting edge protein microarray technology, allowing thousands of parallel assays to detect specific antibodies present in biological specimens, the humoral immune response to entire proteomes of multidrug-resistant bacteria can be profiled, contributing to new vaccine development.

(ix) Target resistant clones and select for drug-susceptible bacteria. A successful effort to select for and increase the reservoir of antibiotic-susceptible bacteria, might ultimately restore, at least partially, the pre-antibiotic landscape. The means to this end is to create conditions that are more favorable for susceptible organisms than to antibiotic-resistant ones. Possible strategies might include antibiotic hybrids (see above), or other approaches that selectively kill a specific class of antibiotic-resistant organisms. Examples include a bactericidal molecule that is activated only after a selective interaction with a $\beta$-lactamase producing bacterium. Screening strategies have shown the possibility of finding compounds that specifically select against resistant bacteria (Stone, et al., 2016), sometimes targeting specific proteins or pathways associated with resistance, e.g. an antibody-drug conjugate that recognizes an antigen unique to the surface of an antibiotic-resistant organism (Mariathasan \& Tan, 2017). Eventually, advanced antibody-drug delivery systems might confer potency to otherwise ineffective antibiotics, such as poorly soluble ones, and then deliver these drugs directly in a strain-specific manner, to antibody-targeted bacteria (Obeid, et al., 2017). Another approach is therapies that specifically decrease the fitness of antibiotic-resistant bacteria, creating a strong and continuous survival disadvantage that eventually pushes them toward extinction. Note that many "high-risk clones" that are antibiotic resistant in humans and animals are well-adapted and highly transmissible commensals (see above). An interesting but potentially risky "ecological" approach is to use genetic engineering to restore susceptibility to antibiotic-resistant bacterial clones, for example using CRISPR-Cas or similar genome editing systems, to re-convert them in antibiotic-susceptible allies (Goren, et al., 2017). 
(x) Modulate the microbiome. The microbiome acts as a main reservoir of antibiotic resistance in/on its host species. In this regard, human and animal opportunistic pathogens (such as intestinal Enterobacterales or Enterococcus) play an important role. Frequently, antibioticresistant commensal bacteria (e.g. $\beta$-lactamases-producing gut Bacteroidetes) degrade antibiotics, thereby reducing the selective power of the drugs and protecting our endogenous microbiota from potential damage. Paradoxically, these organisms create an effective barrier against more widespread resistance in their specific biological niche, which could be regarded as a "resilience mechanism" (Ruppe, et al., 2019) that contributes to "colonization-resistance" against drug-resistant pathogens. For example, under normal conditions, opportunistic pathogens represent less than $1 \%$ of the total intestinal microbiota; however, any dysbiosis and increase in abundance of these opportunists are likely to favor both an increase in resistance and translocation of the pathogens from the intestine to blood, tissues or urine. Hence, the commensal microbiota plays a key role in the fight against the dissemination of resistance. This aspect should be considered before implementing strategies that will selectively eliminate specific antibiotic-resistant bacteria. In fact, fecal microbiota transplantation (FMT) is known to eradicate multidrug-resistant organisms (Gopalsamy, et al., 2018). Phage therapy can be useful to eliminate drug-resistant pathogens as well as opportunistic species that perpetuate dysbiosis and this therapy can also increase colonization resistance (Chan, et al., 2016, Kortright, et al., 2019). Additional strategies for modulating the microbiome include in situ conjugation (Ronda, et al., 2019), optimized use of pre- and/or probiotics, and/or dietary interventions (Goren, et al., 2019) to modify the metabolic landscape of the gut (e.g. short chain fatty-acids).. However, risks associated with some of these microbiome manipulation approaches should not be underestimated (DeFilipp, et al., 2019)..

(xi) Adjuvants, enhancers of antibiotic action. A number of non-conventional antimicrobials might potentiate antibiotic action, narrowing the selective window for resistance (Farha \& Brown, 2013, Brown, 2015). For example, amphiphilic peptides or purified phage lysins permeabilize bacterial cells, which might decrease the survival of low-level resistant mutants, and deplete the population of cells that could subsequently develop mutational or acquired resistance. Additionally, adjuvants may potentiate bacterial killing by increasing the production of harmful reactive oxygen species in the treated bacteria, thereby boosting antibiotic activity. Research is active in the field of antibiotic hybrids, synthetic constructs of antibiotics and antibiotic-enhancers that provide complementary antimicrobial activity (Domalaon, et al., 2018) Thus, adjuvant-antibiotic therapy represents an orthogonal strategy complimentary to the discovery of new antibiotics (Wright, 2016).

(xii) Novel inhibitors of antibiotic resistance. Inhibitors of antibiotic resistance should delay emergence of resistance or restore antibiotic susceptibility. For example, inhibitors, especially when used as a drug-inhibitor combination, can target of enzymes involved in inactivation of antibiotics, such as the $\beta$-lactamases (Docquier \& Mangani, 2018). Indeed, such inhibitors have been used to maintain the therapeutic efficacy of drugs (aminopenicillins, cephalosporins, carbapenems), even though resistance to the inhibitor-drug combination might occur. Research on inhibitors for other drug-modifying proteins, such as aminoglycoside-modifying enzymes, is 
ongoing (Zhu, et al., 2019). Another approach is to inhibit processes leading to resistance, e.g. particular metabolic pathways, membrane transporter enzymes, two-component systems, or efflux pumps (Mahamoud, et al., 2007). Third, disruption of antibiotic resistance genes (see above, CRISPR-Cas-based strategies) to obtain neo-susceptible variants. Fourth, inhibitors of the transfer of MGEs involved in the dissemination of antibiotic resistance, to be used when interbacterial flux of resistance genes is patent (Baquero, et al., 2011), e.g. dissemination of

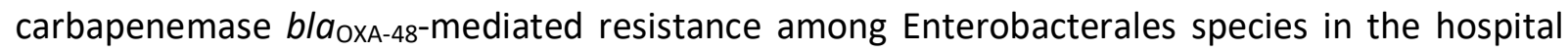
setting (Mairi, et al., 2019).

\section{Patient-level analysis}

\section{Bacterial physiology and within host resilience}

\section{Limitations of antibiotic susceptibility testing}

One question is why susceptible bacteria are resilient to antibiotic treatment when infecting human hosts. It is well documented that the efficacy of antibiotics depends on the physiological state of the bacteria and on the context of exposure. The "textbook" example is penicillin G, which efficiently kills replicating susceptible bacteria but does not kill non-replicating susceptible bacterial cells (Hobby, et al., 1942). This is because penicillin blocks the bacterial cell wall biosynthesis, which is only essential in a dividing cell. In contrast, rifampicin inhibits the bacterial RNA polymerase whether the bacteria are growing or not (Matzura, et al., 1971), with lethal consequences in all bacterial cells. It is therefore not surprising that bacterial susceptibility to antibiotics is strongly affected by the biological context or the conditions that prevail in the laboratory, the clinic or in a human or animal host. Consequently, it is important to be skeptical about the results of standardized laboratory testing for antibiotic sensitivity (Buyck, et al., 2012, Nizet, 2017) and to use caution when inferring susceptibility in an infected patient. We must accept that a measured minimal inhibitory concentration (MIC) for any antibiotic is conditional not absolute. However, in practice, we rely heavily on MICs to prescribe antimicrobials, due to lack of any more relevant pharmacodynamics data. One approach to compensate for this lack of information is to dose the antibiotic significantly higher than the measured MIC, thereby attempting to account for the influence of the physiological state of the bacteria in the host organism. This approach, however, suffers from several problems and several caveats should be stated: 1) the within-host susceptibility spectrum is not well-defined for most antibiotics, 2) the spectrum of bacterial physiologies in the patient is in most cases totally unknown, 3) use of a dose well above the MIC could have undesirable consequences for non-target bacteria in the treated patient, 4) higher doses will result in greater environmental load generated by excretion of excess antibiotic, and 5) very high doses of antibiotics may have paradoxical non-monotonous effects (Eagle effect), and, for many antibiotics (typically beta-lactams), the increase in antibiotic concentration does not produce more killing (Eagle \& Musselman, 1948, Stevens, et al., 1988, Wu, et al., 2015, Jarrad, et al., 2018).

\section{Variation within and between hosts}


As explained above, bacterial responses to antibiotics vary with biological context and physiological state, and in any bacterial population the physiological state of each individual cell varies over time (Brauner, et al., 2016). This is particularly true in the human body, characterized by structural and physiological heterogeneity, in space (different tissues and organs), in time (the process of aging), and in functionality (effect of illnesses, immunology, nutrition). This heterogeneity influences the physiology and phenotype of the target pathogen in unpredictable and continuously changing over the disease course. It is well recognized that human life expectancy has been increasing over the last 150 years, and clinicians may need specific approaches for managing infections in old and very old human patients. Many infections may be more difficult to treat in the elderly, due to a less active immune system and the prevalence of recurring infections with the same clone and certain co-morbidities. Bacteria that persist in patients for an extended period of time often undergo specific adaptive genetic changes that need to be considered when designing a treatment strategy for those patients. In particular, infections caused by opportunistic pathogens that are normally not associated with humans often involve host-adaptive evolutionary processes that are required for prolonged persistence (Figure 3). Such patho-adaptive mutations are usually neglected in the clinic and can result in metabolic and physiological changes (Marvig, et al., 2015).

\section{Low antibiotic concentrations and selection for antibiotic resistance.}

When used to treat a bacterial infection, the antibiotic dose is typically much higher than the MIC, as determined in the laboratory. However, in some body niches, the effective in vivo antibiotic concentration will be lower than average; which could allow bacterial variants with low-level resistance to survive. Such low-level resistant bacteria in the body (see above) increase the risk for subsequent emergence of highly resistant clones, because they will not be considered clinically resistant (resistance level below clinical break-points), and treatment may therefore be continued, increasing the selection pressure towards full resistance (Frimodt-Moller, et al., 2018). Thus, even though antibiotic resistance is carefully monitored and protocols and clinical practice include many steps that guard against its emergence, resistant clones do emerge in patients while they are on antibiotic therapy. Tolerance is the ability of antibiotic-sensitive bacteria to survive when transiently exposed to antibiotic despite having a low MIC for the drug (Brauner, et al., 2016). Under some circumstances, the enhanced survival is attributed to the physiological state of the bacteria, for example dormancy, starvation or reduced metabolism. Tolerance enables bacteria to survive better the duration of bactericidal treatments by delaying the mechanisms that result in death. In addition to being enhanced by some environmental conditions such as starvation, tolerance can also be enhanced by genetic mutations (Handwerger \& Tomasz, 1985), which may arise during treatment (Honsa, et al., 2017). In the laboratory, tolerance can be a step on the path to resistance, and may be important to monitor for its emergence or presence (Levin-Reisman, et al., 2017). Unfortunately, there is no simple assay for measuring bacterial tolerance to antibiotic drugs. So, clinicians can only be aware of antibiotic tolerance as a potential concern that could increase the risk of future antibiotic resistance. Furthermore, often tolerance is the attribute of only a small fraction of the population called persisters (Balaban, et al., 2019) and thus even more difficult to evaluate, a situation that is common in biofilms (see below). 


\section{Focus on improved diagnosis and effective therapeutic approached How can we improve prediction of possible failures in antibiotic therapy?}

It is clear that antibiotic efficacy in vivo in an infected patient is difficult to predict, at least partly because of the impact of bacterial physiological state. Therefore, better methods to predict clinical susceptibility of infecting bacteria to antibiotics are urgently needed. Several factors combine to make this a difficult problem to solve. For example: 1) it may never be possible to identify all factors that critically influence antibiotic susceptibility in the treated patient, and 2) even if an optimal solution can be developed under laboratory conditions, that solution could be too expensive or complicated, or for other reasons impractical to implement in the clinical setting. Nevertheless, it is very important to investigate how and why bacterial susceptibility to antibiotics varies as a function of bacterial growth, metabolic activity and many other features of the bacterial milieu in vivo. Therefore:

(i) For each of the most important bacterial pathogens, a relevant susceptibility matrix should be generated, summarizing the results of a series of MIC determinations under various conditions. Those conditions should test known factors that influence bacterial growth and metabolism, such as nutrient abundance, and reflect the relevant body niche, and any other relevant features of the environment (Kubicek-Sutherland, et al., 2015). It is also important to determine the efficacy of the antibiotics under conditions of slow growth, dormancy, and the lag periods between dormancy and resumption of growth. In these investigations, it is essential to include both reference strains and clinical isolates of the relevant pathogens.

When antibiotic therapy is unsuccessful, even when laboratory tests indicate that the target bacteria are antibiotic-sensitive, it is often postulated that the bacteria have adopted a biofilm lifestyle (Bjarnsholt, et al., 2013). Biofilms are aggregates of bacteria embedded in a polymeric matrix that confers increased tolerance to antibiotics. Biofilms are characterized by spatial heterogeneity, with different bacterial physiologies in different regions of the biofilm. Deeper (less accessible) parts of the biofilm tend to harbor more persister cells. In some infections, the bacteria are located in polymers produced by the host while in others, the bacteria produce their own polymeric matrix. The physiology of the bacteria in such a biofilm is very likely independent of the source of the matrix. It may be possible to detect bacterial biofilms directly, by microscopic inspection of biological samples from the infected patient (see below) (Kragh, et al., 2016), and to develop relatively simple clinical diagnostics for bacterial biofilms.

(ii) Biofilms can be produced in the laboratory using different devices and procedures. These biofilm-like bacterial communities can then be stained and examined under the microscope, to assess whether and how antibiotics gain access to and interact with the embedded bacteria. Interestingly, analyses of the data obtained in biofilm model systems suggest that PK/PD-based dosing of antibiotics might be used to advantage, to increase their antibiotic susceptibility in the infected host. Other advanced human cell culture models, such as bioengineered human tissue cell layers or even organoids, might also be suitable models for better understanding the variable susceptibility of bacteria to antibiotic therapy as a function of biological niche. 
When therapy with the "first-choice" antibiotic is unsuccessful, patients are often treated with a combination of two or more drugs, delivered at the same time or sequentially. It is assumed that the risk that bacteria-resistant to two antibiotics will arise during combination therapy is lower than the risk of single-agent resistance during monotherapy. However, this assumption is often wrong, and as described above for monotherapy, differential compartmentalization of drugs in human organs, tissues, and cells, and other physiological factors can lower susceptibility to a drug combination just as well as to a single drug, with similar impact on therapeutic effect. Therefore, the efficacy of combination therapy is conditional on many unknown in vivo factors, as described above for monotherapy.

(iii) As described above (i and ii), a susceptibility matrix should be generated to help anticipate treatment outcomes for combination antibiotics therapy under variable conditions (see "Emergence, transmission and dissemination of antibiotic resistance at the population level" above). After such data are generated and considered, clinicians can attempt to identify an optimal single or combination antibiotic treatment regimen. These data could also inform consideration of the potential for collateral resistance and sensitivity (see above).

In summary, the main conclusion of the above discussion is that currently available antibiotics, if used wisely and more efficiently will retain the potential to successfully eradicate bacterial pathogens. Furthermore, the risk of promoting emergence of antibiotic-resistant bacteria or persisters can be reduced by using shorter courses of antibiotic therapy. The approach described above is resource- and time-intensive. Therefore, it is essential that all research outcomes and research and clinical data be deposited and stored in publicly-accessible databases. This applies to data on disease/infection progression, treatment and its outcome, genomic information about the infecting bacteria and any other relevant molecular information.

The short-term evaluation of treatment outcomes consists mainly of reporting whether the disease was cured and in how many days. However, it is also important to monitor whether antibiotic-resistant bacteria arise during the course of antibiotic therapy. Consider for example a treatment regimen that cures $70 \%$ of patients in one week, with $30 \%$ of non-responders, all of whom harbor antibiotic-resistant bacteria after treatment. Then, consider a second regimen that cures $50 \%$ of patients in one week, but non-responders do not harbor antibiotic-resistant bacteria. In both cases, the second-line treatment for non-responders would likely be treatment with a different antibiotic. Although $70 \%$ efficacy is preferable to $50 \%$ efficacy in the short term, in the long-term the first treatment should be avoided, because it drives the rapid evolution of resistance and promotes a high rate of antibiotic resistance. Note that in immunocompetent patients, innate immunity by itself is often sufficient to eradicate the pathogen [self-limited infections, see (Levin, et al., 2017)].

\section{Precision medicine and antibiotic resistance}

Generally, short-term antibiotic therapy for an acute infection carries relatively low risk of promoting antibiotic resistance in otherwise healthy (e.g. immunocompetent) individuals. However, the risk increases substantially in immunocompromized patients. For the latter, every 
effort should be made to avoid the "empiric" approach that has promoted overuse of broadspectrum antibiotics and instead the choice of antibiotic needs to be precisely tailored to the individual patient.

\section{Rapid diagnostics}

In general, the choice of the antibiotic for treating a bacterial infection must be based on direct detection of the infecting pathogen and, if possible, its antibiotic susceptibility profile. Thus, in current practice, after a patient is diagnosed, an antimicrobial treatment can typically be selected based on either: A. direct identification of the infecting bacteria and drug resistance markers, which uses mass spectrometry (MALDI-TOF)- and PCR-based detection of molecular biomarkers and allows rapid diagnostics of the pathogen, yet it is still limited to specific biomarkers for a small sub-set of antibiotic resistance genes/gene products; or B. culture-based profiling in which the pathogen is cultivated and assayed for susceptibility to a range of antibiotics. Unfortunately, the latter method is inherently slow and limited to those microbes that grow at a reasonably fast rate in the laboratory. However, some culture media can "wake up" dormant persisters and fastidious bacteria, promote faster growth, and the sensitivity of detection can be enhanced, which can be further improved by using e.g. microfluidics (<30min from sample to answer) (Baltekin, et al., 2017). In many infections, the pathogen is a single entity, and diagnostics are usually based on the analysis of a single clinical isolate. However, patients often have mixed infections and are colonized by diverse bacterial populations in their microbiota. Current diagnostics will commonly miss the relevant drug resistance profile within the pathogen population, which can lead to selection of an ineffective and inappropriate antibiotic therapy.

\section{"Omics"-based diagnostics in precision medicine}

Genomics-based approaches are useful for broad identification of antibiotic resistance genes and alleles. Indeed, with ever improving "omics", bioinformatics and machine learning approaches, one can foresee in the future an integrated solution capable of identifying known as well as putative antibiotic resistance genes (Ruppe, et al., 2019). Indeed, we envision that within a decade, whole genome sequencing (WGS) will become standard for pathogen identification and characterization, at least for severe cases like blood infections that require rapid and accurate identification of resistance genes. Already, WGS is highly effective and informative and a prime tool for identifying the chromosomal antibiotic resistance signatures in genomic DNA of the slowgrowing clonal Mycobacterium tuberculosis (Cohen, et al., 2019, Gygli, et al., 2019). Omics techniques are currently most relevant in surveillance by molecular epidemiology. However, for diagnostic purposes there are still some challenges: Current WGS technology still requires cultivation of the clinical specimens and isolation of the pathogen, unless metagenomic studies are performed. Even though DNA isolation and sequencing technologies can be performed within hours, the bioinformatics analysis is still a more time-consuming bottle-neck. For precision medicine to be a reality, diagnosis should be made in a few (2-3) hours, without microbe isolation. For rapid WGS of a complex mixed biological sample (e.g. metagenomics analysis), there is urgent need for novel technologies, capable of rapidly providing clinically-actionable data on bacterial species and their antibiotic resistance signatures. Such technologies should have a low rate of false positive or negative results, which can lead to incorrect diagnosis and treatment choices. 
Recent advances in "omics" technologies and informatics could soon potentially transform and dramatically improve our understanding of AMR. Specifically, WGS methods such as Nanopore and PacBio sequencing not only have very long reads but can also reveal DNA (and in the case of Nanopore also RNA) modifications (Hofer \& Jaschke, 2018), such as methylation (Casadesus \& Low, 2006, Brockman, et al., 2018). DNA methylation can indeed produce population heterogeneity and epigenetic effects on gene expression, promoting adaptive resistance in genetically susceptible bacteria (Motta, et al., 2015). Similarly, post-translational modification of proteins, which could play a role in antibiotic resistance, can be identified by advanced proteomics (Birhanu, et al., 2017, Birhanu, et al., 2019). Epigenetics, epitranscriptomics and epiproteomics are thus expected to unveil novel aspects of the complexity of antibiotic resistance as current efforts proceed (Chen, et al., 2018). Furthermore, application of machine learning approaches to omics data can identify gene expression signatures, other molecular motifs, possibly leading to biomarkers and novel diagnostics for antibiotic resistance. We envision that these methods will, in the near future, transform the detection, diagnostics and treatment of infectious disease, supporting highly-tailored and much more effective treatment. Ultimately, a multifaceted "omics" approach that integrates comprehensive pathogen and antibiotic resistance profiling with personalized patient data such as natural microbiota composition (metagenomics), genetic predisposition to infections, and immune response to infection together with PK/PD modeling, has the potential to enable optimized precision medicine for antimicrobial prescribing practices. Importantly, such comprehensive analysis must be accurate, to avoid directing the therapy toward contaminants and harmless colonizers. Whether based on cultivation- or biomarker-based antimicrobial susceptibility data, strong bactericidal agents at the appropriate doses (the steeper the Hill function of the killing curve, the better) should be used. In immunocompromized patients, use of dosage schedules, and more frequent use of antibiotic combinations that have been tested and shown to be synergistic, is advised, potentially along with measures to prevent overgrowth of opportunistic antibiotic-resistant pathogens in the gut, by use of drug-inactivating or antibiotic-adsorbing compounds (de Gunzburg, et al., 2015). Such compounds can also have the added benefit of reducing antibiotic excretion by the patients.

\section{Concluding words}

The oft-repeated mantra "prudent use of antibiotics" or "les antibiotiques c'est pas automatiques" reflect the belief that any unjustified increase in antibiotic use will increase the prevalence of AMR. This is a certain fact, one that has been demonstrated at the individual, hospital, farm, national and global environmental levels (Goossens, et al., 2005). To us the most important potential problem at the global scale is the cumulative "antibiotic pollution" of the environment, followed by "antibiotic-resistance pollution" of the genomes of all living organisms (Berendonk, et al., 2015). As for global climate change, pollution at this scale may be difficult or impossible to revert, leading to irreversible and devastating consequences on environmental and human health. However, there is still hope, if improved global sanitation, and advanced techniques for water and sludge treatment (Zheng, et al., 2019) prove adequate to attenuate further emergence and dissemination of antibiotic resistance. Global problems require global solutions and only a concerted and sustained international effort can succeed in reversing the ongoing trajectories and trends. Sanitation and health management practice worldwide, in 
poorer and more affluent countries and communities alike, will need to improve. Therefore, it is in the direct interest of affluent nations to widely share and leverage all available resources on a global scale, because this is our most promising approach for winning the global war against antimicrobial resistance.

\section{BOX Rapid diagnostics for antibiotic resistance}

\section{Point-of-care (POC) diagnostics - Current and future challenges}

POC testing as defined by ISO 22870: 2016 is testing that is performed near or at the site of patient care, so that patient-specific test results can immediately inform clinical care decisions for that patient. Better antibiotic stewardship requires access to improved, faster and affordable diagnostics for infectious disease and antibiotic resistance. Rapid diagnostics are analyses that require a "short time" ( $<1$ - a few hours), and include analyses performed both as "patient-near analysis (PNA)" and in the clinical laboratory. PNA for infectious disease is performed by healthcare personnel near the patient and is based mostly on immunological and real-time PCR assays. The results are obtained quickly and can often be communicated directly to the patient, if the medical personnel have sufficient experience in assessing and interpreting test results. In contrast to most PNA, POC laboratories often operate $24 / 7$ to provide diagnoses in less than 2 hours, and are operated by specifically-trained staff. In general, POC assays reduce turn-aroundtime significantly (typically, 15 min to three hours), while the performance remains comparable to that obtained in a clinical laboratory. In the near future, multiple POC platforms will, after appropriate validation, become commercially available. However, increased reliance on POC laboratories should not lead to reduced national surveillance of selected infections, such as emerging pathogens and Mycobacterium tuberculosis.

Rapid diagnostic microbiology tests generally involve direct detection of microbial proteins or nucleic acids and is routinely performed in a clinical laboratory. In contrast, reliable detection of emerging resistance will require novel diagnostics. Commercial nucleic-acid based tests currently exist for identification of $v a n A$ / vanB genes in vancomycin-resistant enterococci or staphylococci, and for extended spectrum $\beta$-lactamases (for example CTX-M-1, CTX-M-9, and NDM). These tests can also be applied to culture from solid media or directly to positive blood cultures in 20-30 minutes. Some current methods that involve PCR directly from blood, and subsequent hybridization to nanoparticles and detection by magnetic resonance can obtain a sensitivity down to $1 \mathrm{cfu} / \mathrm{mL}$, with a specificity of about $99 \%$, but the time for detection after sampling is approximately 4 hours. More rapid analysis of blood can be obtained during suspected bacteremia using MALDI-TOF mass spectrometry. It remains to be seen whether newer methods, such as biotyping based on infra-red spectroscopy will also become clinically applicable in the near future. Importantly, most of the cutting-edge technologies require expensive state-of-theart equipment, but some, such as isothermal amplification, DNA Endonuclease-Targeted CRISPR Trans Reporter (DETECTR) and Specific High-Sensitivity Enzymatic Reporter UnLOCKing 
(SHERLOCK) can also be used in developing countries or other resource-poor settings (Petri \& Pattanayak, 2018).

\section{Acknowledgments.}

This work was supported by the FEMS European Academy of Microbiology. The authors thank Eliora Z. Ron for organizing this working group and for contributing to the discussions that led to the writing of this manuscript. We also thank Netta Shemesh for generating two of the illustrations.

Legends

Figure 1. Selective pressures of varying strength in humans, animals and the environment Figure 2. Dissemination of antibiotic resistance in bacteria

Figure 3. Different modes of survival under antibiotic treatments: Resistance is due to increased expression of resistance factors that decrease the effective concentration of antibiotic such as efflux pumps, target with modified affinity, decreased permeability, etc. It is typically acquired by mutations or Horizontal Gene Transfer (HGT), but can also triggered by environmental signals. Heterogeneity of the expression of the resistance factors can lead to heteroresistance. Tolerance is due to decreased kill rate under antibiotics due to a slowing down of processes in bacteria. It may be acquired by genetic factors or triggered by environmental conditions. Heterogeneity of the tolerance phenotype leads to antibiotic persistence i.e. when tolerance occurs in a subpopulation within the clonal population.

\section{References}

Abubucker S, Segata N, Goll J, et al. (2012) Metabolic reconstruction for metagenomic data and its application to the human microbiome. PLoS Comput Biol 8: e1002358.

Al-Hamad A, Burnie J \& Upton M (2011) Enhancement of antibiotic susceptibility of Stenotrophomonas maltophilia using a polyclonal antibody developed against an ABC multidrug efflux pump. Can J Microbiol 57: 820-828.

Andersson DI (2015) Improving predictions of the risk of resistance development against new and old antibiotics. Clin Microbiol Infect 21: 894-898.

Andersson DI \& Hughes D (2010) Antibiotic resistance and its cost: is it possible to reverse resistance? Nat Rev Microbiol 8: 260-271.

Balaban NQ, Helaine S, Lewis K, et al. (2019) Definitions and guidelines for research on antibiotic persistence. Nat Rev Microbiol 17: 441-448.

Baltekin Ö, Boucharin A, Tano E, Andersson DI \& Elf J (2017) Antibiotic susceptibility testing in less than 30 min using direct single-cell imaging. Proc Natl Acad Sci U S A 114: 9170-9175. 
Baquero F (2007) Evaluation of Risks and Benefits of Consumption of Antibiotics: From Individual to Public Health. Encyclopedia of Infectious Diseases: Modern Methodologies,(Tibayrenc M, ed.^eds.), p.^pp. 509- 516. John Wiley and Sons.

Baquero F \& Negri MC (1997) Selective compartments for resistant microorganisms in antibiotic gradients. Bioessays 19: 731-736.

Baquero F, Vicente MF \& Perez-Diaz JC (1985) beta-Lactam coselection of sensitive and TEM-1 beta-lactamase-producing subpopulations in heterogeneous Escherichia coli colonies. $J$ Antimicrob Chemother 15: 151-157.

Baquero F, Coque TM \& de la Cruz F (2011) Ecology and evolution as targets: the need for novel eco-evo drugs and strategies to fight antibiotic resistance. Antimicrob Agents Chemother 55: 36493660 .

Baquero F, Lanza VF, Canton R \& Coque TM (2015) Public health evolutionary biology of antimicrobial resistance: priorities for intervention. Evol Appl 8: 223-239.

Baquero F, Coque TM, Martinez JL, Aracil S \& Lanza VF (In press) Gene Transmission in the One Health Microbiosphere and the Channels of Antimicrobial Resistance. Front Microbiol.

Baur D, Gladstone BP, Burkert F, Carrara E, Foschi F, Dobele S \& Tacconelli E (2017) Effect of antibiotic stewardship on the incidence of infection and colonisation with antibiotic-resistant bacteria and Clostridium difficile infection: a systematic review and meta-analysis. Lancet Infect Dis 17: 990-1001.

Begier E, Seiden DJ, Patton M, et al. (2017) SA4Ag, a 4-antigen Staphylococcus aureus vaccine, rapidly induces high levels of bacteria-killing antibodies. Vaccine 35: 1132-1139.

Bengtsson-Palme J, Milakovic M, Svecova H, Ganjto M, Jonsson V, Grabic R \& Udikovic-Kolic $\mathrm{N}$ (2019) Industrial wastewater treatment plant enriches antibiotic resistance genes and alters the structure of microbial communities. Water Res 162: 437-445.

Berendonk TU, Manaia CM, Merlin C, et al. (2015) Tackling antibiotic resistance: the environmental framework. Nat Rev Microbiol 13: 310-317.

Bergstrom CT, Lo M \& Lipsitch M (2004) Ecological theory suggests that antimicrobial cycling will not reduce antimicrobial resistance in hospitals. Proc Natl Acad Sci U S A 101: 13285-13290. Birhanu AG, Yimer SA, Holm-Hansen C, Norheim G, Aseffa A, Abebe M \& Tonjum T (2017) $\mathrm{N}$ - and O-Acetylation in Mycobacterium tuberculosis Lineage 7 and Lineage 4 Strains: Proteins Involved in Bioenergetics, Virulence, and Antimicrobial Resistance Are Acetylated. J Proteome Res 16: 4045-4059.

Birhanu AG, Yimer SA, Kalayou S, et al. (2019) Ample glycosylation in membrane and cell envelope proteins may explain the phenotypic diversity and virulence in the Mycobacterium tuberculosis complex. Sci Rep 9: 2927.

Bjarnsholt T, Ciofu O, Molin S, Givskov M \& Hoiby N (2013) Applying insights from biofilm biology to drug development - can a new approach be developed? Nat Rev Drug Discov 12: 791 808.

Bjorkman J, Nagaev I, Berg OG, Hughes D \& Andersson DI (2000) Effects of environment on compensatory mutations to ameliorate costs of antibiotic resistance. Science 287: 1479-1482.

Boxall AB (2004) The environmental side effects of medication. EMBO Rep 5: 1110-1116.

Branche A, Neeser O, Mueller B \& Schuetz P (2019) Procalcitonin to guide antibiotic decision making. Curr Opin Infect Dis 32: 130-135.

Brauner A, Fridman O, Gefen O \& Balaban NQ (2016) Distinguishing between resistance, tolerance and persistence to antibiotic treatment. Nat Rev Microbiol 14: 320-330. 
Brockman KL, Azzari PN, Branstool MT, et al. (2018) Epigenetic Regulation Alters Biofilm Architecture and Composition in Multiple Clinical Isolates of Nontypeable Haemophilus influenzae. MBio 9.

Brown D (2015) Antibiotic resistance breakers: can repurposed drugs fill the antibiotic discovery void? Nat Rev Drug Discov 14: 821-832.

Buyck JM, Plesiat P, Traore H, Vanderbist F, Tulkens PM \& Van Bambeke F (2012) Increased susceptibility of Pseudomonas aeruginosa to macrolides and ketolides in eukaryotic cell culture media and biological fluids due to decreased expression of oprM and increased outer-membrane permeability. Clin Infect Dis 55: 534-542.

Cairns J, Ruokolainen L, Hultman J, Tamminen M, Virta M \& Hiltunen T (2018) Ecology determines how low antibiotic concentration impacts community composition and horizontal transfer of resistance genes. Commun Biol 1: 35.

Casadesus J \& Low D (2006) Epigenetic gene regulation in the bacterial world. Microbiol Mol Biol Rev 70: 830-856.

Chan BK, Brown K, Kortright KE, Mao S \& Turner PE (2016) Extending the lifetime of antibiotics: how can phage therapy help? Future Microbiol 11: 1105-1107.

Chen L, Li H, Chen T, et al. (2018) Genome-wide DNA methylation and transcriptome changes in Mycobacterium tuberculosis with rifampicin and isoniazid resistance. Int J Clin Exp Pathol 11: 3036-3045.

Cirz RT, Chin JK, Andes DR, de Crecy-Lagard V, Craig WA \& Romesberg FE (2005) Inhibition of mutation and combating the evolution of antibiotic resistance. PLoS Biol 3: e176.

Collignon P, Beggs JJ, Walsh TR, Gandra S \& Laxminarayan R (2018) Anthropological and socioeconomic factors contributing to global antimicrobial resistance: a univariate and multivariable analysis. Lancet Planet Health 2: e398-e405.

Courvalin P (2006) Vancomycin resistance in gram-positive cocci. Clin Infect Dis 42 Suppl 1: S25-34.

Cussotto S, Clarke G, Dinan TG \& Cryan JF (2019) Psychotropics and the Microbiome: a Chamber of Secrets. Psychopharmacology (Berl) 236: 1411-1432.

Da Cunha V, Davies MR, Douarre PE, et al. (2014) Streptococcus agalactiae clones infecting humans were selected and fixed through the extensive use of tetracycline. Nat Commun 5: 4544.

de Gunzburg J, Ducher A, Modess C, et al. (2015) Targeted adsorption of molecules in the colon with the novel adsorbent-based medicinal product, DAV132: A proof of concept study in healthy subjects. J Clin Pharmacol 55: 10-16.

de Visser JA \& Krug J (2014) Empirical fitness landscapes and the predictability of evolution. Nat Rev Genet 15: 480-490.

DeNegre AA, Ndeffo Mbah ML, Myers K \& Fefferman NH (2019) Emergence of antibiotic resistance in immunocompromised host populations: A case study of emerging antibiotic resistant tuberculosis in AIDS patients. PLoS One 14: e0212969.

Dethlefsen L \& Relman DA (2011) Incomplete recovery and individualized responses of the human distal gut microbiota to repeated antibiotic perturbation. Proc Natl Acad Sci U S A $\mathbf{1 0 8}$ Suppl 1: 4554-4561.

Docquier JD \& Mangani S (2018) An update on beta-lactamase inhibitor discovery and development. Drug Resist Updat 36: 13-29.

Domalaon R, Idowu T, Zhanel GG \& Schweizer F (2018) Antibiotic Hybrids: the Next Generation of Agents and Adjuvants against Gram-Negative Pathogens? Clin Microbiol Rev 31. 
Dorado-García A, Heederik DJ, Jacobs JJH, Mevius DJ, Wagenaar JA, Van Geijlswijk IM \& Mouton JW (2016) Quantitative assessment of antimicrobial resistance in livestock during the course of a nationwide antimicrobial use reduction in the Netherlands. Journal of Antimicrobial Chemotherapy 71: 3607-3619.

Dortet L, Glaser P, Kassis-Chikhani N, et al. (2018) Long-lasting successful dissemination of resistance to oxazolidinones in MDR Staphylococcus epidermidis clinical isolates in a tertiary care hospital in France. J Antimicrob Chemother 73: 41-51.

Drlica K \& Zhao X (2007) Mutant selection window hypothesis updated. Clin Infect Dis 44: 681688.

Durão P, Balbontín R \& Gordo I (2018) Evolutionary Mechanisms Shaping the Maintenance of Antibiotic Resistance. Trends in Microbiology 26: 677-691.

Eagle H \& Musselman AD (1948) The rate of bactericidal action of penicillin in vitro as a function of its concentration, and its paradoxically reduced activity at high concentrations against certain organisms. J Exp Med 88: 99-131.

Farha MA \& Brown ED (2013) Discovery of antibiotic adjuvants. Nat Biotechnol 31: 120-122.

Flach CF, Pal C, Svensson CJ, et al. (2017) Does antifouling paint select for antibiotic resistance? Sci Total Environ 590-591: 461-468.

Forsberg KJ, Reyes A, Wang B, Selleck EM, Sommer MO \& Dantas G (2012) The shared antibiotic resistome of soil bacteria and human pathogens. Science 337: 1107-1111.

Frana TS, Beahm AR, Hanson BM, et al. (2013) Isolation and characterization of methicillinresistant Staphylococcus aureus from pork farms and visiting veterinary students. PLoS One 8: e53738.

Frimodt-Moller J, Rossi E, Haagensen JAJ, Falcone M, Molin S \& Johansen HK (2018) Mutations causing low level antibiotic resistance ensure bacterial survival in antibiotic-treated hosts. Sci Rep 8: 12512 .

Gatica J \& Cytryn E (2013) Impact of treated wastewater irrigation on antibiotic resistance in the soil microbiome. Environ Sci Pollut Res Int 20: 3529-3538.

Gerding DN, Larson TA, Hughes RA, Weiler M, Shanholtzer C \& Peterson LR (1991) Aminoglycoside resistance and aminoglycoside usage: ten years of experience in one hospital. Antimicrob Agents Chemother 35: 1284-1290.

Getino M \& de la Cruz F (2018) Natural and Artificial Strategies To Control the Conjugative Transmission of Plasmids. Microbiol Spectr $\mathbf{6}$.

Gonzales PR, Pesesky MW, Bouley R, et al. (2015) Synergistic, collaterally sensitive beta-lactam combinations suppress resistance in MRSA. Nat Chem Biol 11: 855-861.

Goossens H, Ferech M, Vander Stichele R \& Elseviers M (2005) Outpatient antibiotic use in Europe and association with resistance: a cross-national database study. Lancet 365: 579-587.

Gopalsamy SN, Woodworth MH, Wang T, et al. (2018) The Use of Microbiome Restoration Therapeutics to Eliminate Intestinal Colonization With Multidrug-Resistant Organisms. Am J Med Sci 356: 433-440.

Goren I, Godny L, Reshef L, Yanai H, Gophna U, Tulchinsky H \& Dotan I (2019) Starch Consumption May Modify Antiglycan Antibodies and Fecal Fungal Composition in Patients With Ileo-Anal Pouch. Inflamm Bowel Dis 25: 742-749.

Goren M, Yosef I \& Qimron U (2017) Sensitizing pathogens to antibiotics using the CRISPR-Cas system. Drug Resist Updat 30: 1-6. 
Gullberg E, Albrecht LM, Karlsson C, Sandegren L \& Andersson DI (2014) Selection of a multidrug resistance plasmid by sublethal levels of antibiotics and heavy metals. MBio 5: e0191801914.

Gullberg E, Cao S, Berg OG, Ilbäck C, Sandegren L, Hughes D \& Andersson DI (2011) Selection of Resistant Bacteria at Very Low Antibiotic Concentrations. PLOS Pathogens 7: e1002158.

Haber M, Levin BR \& Kramarz P (2010) Antibiotic control of antibiotic resistance in hospitals: a simulation study. BMC Infect Dis 10: 254.

Handwerger S \& Tomasz A (1985) Antibiotic Tolerance Among Clinical Isolates of Bacteria. Annual Review of Pharmacology and Toxicology 25: 349-380.

Hernando-Amado S, Coque TM, Baquero F \& Martinez JL (2019) Defining and combating antibiotic resistance from One Health and Global Health perspectives. Nat Microbiol 4: 14321442.

Hiltunen T, Cairns J, Frickel J, et al. (2018) Dual-stressor selection alters eco-evolutionary dynamics in experimental communities. Nat Ecol Evol 2: 1974-1981.

Hobby GL, Meyer K \& Chaffee E (1942) Observations on the Mechanism of Action of Penicillin. Proceedings of the Society for Experimental Biology and Medicine 50: 281-285.

Hofer K \& Jaschke A (2018) Epitranscriptomics: RNA Modifications in Bacteria and Archaea. Microbiol Spectr 6.

Honsa ES, Cooper VS, Mhaissen MN, et al. (2017) RelA Mutant Enterococcus faecium with Multiantibiotic Tolerance Arising in an Immunocompromised Host. MBio 8.

Hughes D \& Andersson DI (2012) Selection of resistance at lethal and non-lethal antibiotic concentrations. Curr Opin Microbiol 15: 555-560.

Hughes D \& Andersson DI (2017) Environmental and genetic modulation of the phenotypic expression of antibiotic resistance. FEMS Microbiol Rev 41: 374-391.

Hughes D \& Andersson DI (2017) Evolutionary Trajectories to Antibiotic Resistance. Annu Rev Microbiol 71: 579-596.

Huseby DL, Pietsch F, Brandis G, Garoff L, Tegehall A \& Hughes D (2017) Mutation Supply and Relative Fitness Shape the Genotypes of Ciprofloxacin-Resistant Escherichia coli. Mol Biol Evol 34: 1029-1039.

Huttner A \& Gambillara V (2018) The development and early clinical testing of the ExPEC4V conjugate vaccine against uropathogenic Escherichia coli. Clin Microbiol Infect 24: 1046-1050.

Imamovic L \& Sommer MOA (2013) Use of Collateral Sensitivity Networks to Design Drug Cycling Protocols That Avoid Resistance Development. Sci Transl Med 5: 204ra132-204ra132.

Jansen KU, Knirsch C \& Anderson AS (2018) The role of vaccines in preventing bacterial antimicrobial resistance. Nat Med 24: 10-19.

Jarrad AM, Blaskovich MAT, Prasetyoputri A, Karoli T, Hansford KA \& Cooper MA (2018) Detection and Investigation of Eagle Effect Resistance to Vancomycin in Clostridium difficile With an ATP-Bioluminescence Assay. Front Microbiol 9: 1420.

Kallonen T, Brodrick HJ, Harris SR, et al. (2017) Systematic longitudinal survey of invasive Escherichia coli in England demonstrates a stable population structure only transiently disturbed by the emergence of ST131. Genome Res.

Kantele A, Pakkanen SH, Siitonen A, Karttunen R \& Kantele JM (2012) Live oral typhoid vaccine Salmonella Typhi Ty21a - a surrogate vaccine against non-typhoid salmonella? Vaccine 30: 72387245.

Karkman A, Parnanen K \& Larsson DGJ (2019) Fecal pollution can explain antibiotic resistance gene abundances in anthropogenically impacted environments. Nat Commun 10: 80. 
Kash JC \& Taubenberger JK (2015) The role of viral, host, and secondary bacterial factors in influenza pathogenesis. Am J Pathol 185: 1528-1536.

Kim S, Lieberman TD \& Kishony R (2014) Alternating antibiotic treatments constrain evolutionary paths to multidrug resistance. Proceedings of the National Academy of Sciences 111: 14494-14499.

Knopp M \& Andersson DI (2015) Amelioration of the Fitness Costs of Antibiotic Resistance Due To Reduced Outer Membrane Permeability by Upregulation of Alternative Porins. Mol Biol Evol 32: 3252-3263.

Knopp M \& Andersson DI (2018) Predictable Phenotypes of Antibiotic Resistance Mutations. MBio 9.

Ko HHT, Lareu RR, Dix BR \& Hughes JD (2017) Statins: antimicrobial resistance breakers or makers? PeerJ 5: e3952.

Kortright KE, Chan BK, Koff JL \& Turner PE (2019) Phage Therapy: A Renewed Approach to Combat Antibiotic-Resistant Bacteria. Cell Host Microbe 25: 219-232.

Kragh KN, Hutchison JB, Melaugh G, et al. (2016) Role of Multicellular Aggregates in Biofilm Formation. MBio 7: e00237.

Kubicek-Sutherland JZ, Heithoff DM, Ersoy SC, et al. (2015) Host-dependent Induction of Transient Antibiotic Resistance: A Prelude to Treatment Failure. EBioMedicine 2: 1169-1178.

Kwong JC, Maaten S, Upshur RE, Patrick DM \& Marra F (2009) The effect of universal influenza immunization on antibiotic prescriptions: an ecological study. Clin Infect Dis 49: 750-756.

Lakin SM, Dean C, Noyes NR, et al. (2017) MEGARes: an antimicrobial resistance database for high throughput sequencing. Nucleic Acids Res 45: D574-D580.

Lanza VF, Baquero F, Martinez JL, et al. (2018) In-depth resistome analysis by targeted metagenomics. Microbiome 6: 11 .

Lazar V, Pal Singh G, Spohn R, et al. (2013) Bacterial evolution of antibiotic hypersensitivity. Mol Syst Biol 9: 700.

Lazar V, Nagy I, Spohn R, et al. (2014) Genome-wide analysis captures the determinants of the antibiotic cross-resistance interaction network. Nat Commun 5: 4352.

Lazar V, Martins A, Spohn R, et al. (2018) Antibiotic-resistant bacteria show widespread collateral sensitivity to antimicrobial peptides. Nat Microbiol 3: 718-731.

Levin-Reisman I, Ronin I, Gefen O, Braniss I, Shoresh N \& Balaban NQ (2017) Antibiotic tolerance facilitates the evolution of resistance. Science 355: 826-830.

Levin BR, Baquero F, Ankomah PP \& McCall IC (2017) Phagocytes, Antibiotics, and SelfLimiting Bacterial Infections. Trends Microbiol 25: 878-892.

Lipsitch M, Bergstrom CT \& Levin BR (2000) The epidemiology of antibiotic resistance in hospitals: paradoxes and prescriptions. Proc Natl Acad Sci U S A 97: 1938-1943.

Liu A, Fong A, Becket E, et al. (2011) Selective advantage of resistant strains at trace levels of antibiotics: a simple and ultrasensitive color test for detection of antibiotics and genotoxic agents. Antimicrob Agents Chemother 55: 1204-1210.

Liu YY, Wang Y, Walsh TR, et al. (2016) Emergence of plasmid-mediated colistin resistance mechanism MCR-1 in animals and human beings in China: a microbiological and molecular biological study. Lancet Infect Dis 16: 161-168.

Low M, Almog R, Balicer RD, Liberman N, Raz R, Peretz A \& Nitzan O (2018) Infectious disease burden and antibiotic prescribing in primary care in Israel. Ann Clin Microbiol Antimicrob 17: 26. 
Ludden C, Raven KE, Jamrozy D, et al. (2019) One Health Genomic Surveillance of Escherichia coli Demonstrates Distinct Lineages and Mobile Genetic Elements in Isolates from Humans versus Livestock. MBio 10.

Lundstrom SV, Ostman M, Bengtsson-Palme J, et al. (2016) Minimal selective concentrations of tetracycline in complex aquatic bacterial biofilms. Sci Total Environ 553: 587-595.

Mahamoud A, Chevalier J, Alibert-Franco S, Kern WV \& Pages JM (2007) Antibiotic efflux pumps in Gram-negative bacteria: the inhibitor response strategy. J Antimicrob Chemother 59: 1223-1229.

Maillard JY (2018) Resistance of Bacteria to Biocides. Microbiol Spectr 6.

Mairi A, Pantel A, Ousalem F, Sotto A, Touati A \& Lavigne JP (2019) OXA-48-producing Enterobacterales in different ecological niches in Algeria: clonal expansion, plasmid characteristics and virulence traits. J Antimicrob Chemother 74: 1848-1855.

Marcusson LL, Frimodt-Moller N \& Hughes D (2009) Interplay in the selection of fluoroquinolone resistance and bacterial fitness. PLoS Pathog 5: e1000541.

Mariathasan S \& Tan MW (2017) Antibody-Antibiotic Conjugates: A Novel Therapeutic Platform against Bacterial Infections. Trends Mol Med 23: 135-149.

Martinez JL, Coque TM \& Baquero F (2015) What is a resistance gene? Ranking risk in resistomes. Nat Rev Microbiol 13: 116-123.

Marvig RL, Sommer LM, Molin S \& Johansen HK (2015) Convergent evolution and adaptation of Pseudomonas aeruginosa within patients with cystic fibrosis. Nat Genet 47: 57-64.

Masters PA, O'Bryan TA, Zurlo J, Miller DQ \& Joshi N (2003) Trimethoprim-sulfamethoxazole revisited. Arch Intern Med 163: 402-410.

Matzura H, Molin S \& Maaloe O (1971) Sequential biosynthesis of the and ' subunits of the DNAdependent RNA polymerase from Escherichia coli. J Mol Biol 59: 17-25.

Mebis J, Goossens H, Bruyneel P, et al. (1998) Decreasing antibiotic resistance of Enterobacteriaceae by introducing a new antibiotic combination therapy for neutropenic fever patients. Leukemia 12: 1627-1629.

Micenkova L, Bosak J, Staudova B, et al. (2016) Microcin determinants are associated with B2 phylogroup of human fecal Escherichia coli isolates. Microbiologyopen 5: 490-498.

Motta SS, Cluzel P \& Aldana M (2015) Adaptive resistance in bacteria requires epigenetic inheritance, genetic noise, and cost of efflux pumps. PLoS One 10: e0118464.

Moura de Sousa J, Balbontín R, Durão P \& Gordo I (2017) Multidrug-resistant bacteria compensate for the epistasis between resistances. PLoS Biol 15: e2001741.

Mughini-Gras L, Dorado-García A, van Duijkeren E, et al. (2019) Attributable sources of community-acquired carriage of Escherichia coli containing $\beta$-lactam antibiotic resistance genes: a population-based modelling study. The Lancet Planetary Health 3: e357-e369.

Munck C, Gumpert HK, Wallin AI, Wang HH \& Sommer MO (2014) Prediction of resistance development against drug combinations by collateral responses to component drugs. Sci Transl Med 6: 262 ra156.

Nemeth J, Oesch G \& Kuster SP (2015) Bacteriostatic versus bactericidal antibiotics for patients with serious bacterial infections: systematic review and meta-analysis. $J$ Antimicrob Chemother 70: $382-395$.

Nichol D, Rutter J, Bryant C, et al. (2019) Antibiotic collateral sensitivity is contingent on the repeatability of evolution. Nat Commun 10: 334. 
Nicoloff H \& Andersson DI (2016) Indirect resistance to several classes of antibiotics in cocultures with resistant bacteria expressing antibiotic-modifying or -degrading enzymes. J Antimicrob Chemother 71: 100-110.

Nizet V (2017) The Accidental Orthodoxy of Drs. Mueller and Hinton. EBioMedicine 22: 26-27. Obeid MA, Al Qaraghuli MM, Alsaadi M, Alzahrani AR, Niwasabutra K \& Ferro VA (2017) Delivering natural products and biotherapeutics to improve drug efficacy. Ther Deliv 8: 947-956. Oliver A, Canton R, Campo P, Baquero F \& Blazquez J (2000) High frequency of hypermutable Pseudomonas aeruginosa in cystic fibrosis lung infection. Science 288: 1251-1254.

Oz T, Guvenek A, Yildiz S, et al. (2014) Strength of selection pressure is an important parameter contributing to the complexity of antibiotic resistance evolution. Mol Biol Evol 31: 2387-2401.

Pal C, Bengtsson-Palme J, Kristiansson E \& Larsson DG (2015) Co-occurrence of resistance genes to antibiotics, biocides and metals reveals novel insights into their co-selection potential. $B M C$ Genomics 16: 964.

Perichon B \& Courvalin P (2009) VanA-type vancomycin-resistant Staphylococcus aureus. Antimicrob Agents Chemother 53: 4580-4587.

Petri K \& Pattanayak V (2018) SHERLOCK and DETECTR Open a New Frontier in Molecular Diagnostics. CRISPR J 1: 209-211.

Planet PJ, LaRussa SJ, Dana A, et al. (2013) Emergence of the epidemic methicillin-resistant Staphylococcus aureus strain USA300 coincides with horizontal transfer of the arginine catabolic mobile element and speG-mediated adaptations for survival on skin. MBio 4: e00889-00813.

Podnecky NL, Fredheim EGA, Kloos J, et al. (2018) Conserved collateral antibiotic susceptibility networks in diverse clinical strains of Escherichia coli. Nat Commun 9: 3673.

Price LB, Johnson JR, Aziz M, et al. (2013) The epidemic of extended-spectrum-beta-lactamaseproducing Escherichia coli ST131 is driven by a single highly pathogenic subclone, H30-Rx. MBio 4: e00377-00313.

Price LB, Stegger M, Hasman H, et al. (2012) Staphylococcus aureus CC398: host adaptation and emergence of methicillin resistance in livestock. MBio 3.

Ragheb MN, Thomason MK, Hsu C, et al. (2019) Inhibiting the Evolution of Antibiotic Resistance. Mol Cell 73: 157-165 e155.

Rinsky JL, Nadimpalli M, Wing S, et al. (2013) Livestock-associated methicillin and multidrug resistant Staphylococcus aureus is present among industrial, not antibiotic-free livestock operation workers in North Carolina. PLoS One 8: e67641.

Roemhild R, Gokhale CS, Dirksen P, et al. (2018) Cellular hysteresis as a principle to maximize the efficacy of antibiotic therapy. Proceedings of the National Academy of Sciences 115: 97679772.

Ronda C, Chen SP, Cabral V, Yaung SJ \& Wang HH (2019) Metagenomic engineering of the mammalian gut microbiome in situ. Nat Methods 16: 167-170.

Rosenkilde CEH, Munck C, Porse A, Linkevicius M, Andersson DI \& Sommer MOA (2019) Collateral sensitivity constrains resistance evolution of the CTX-M-15 beta-lactamase. Nat Commun 10: 618.

Ruppe E, Ghozlane A, Tap J, et al. (2019) Prediction of the intestinal resistome by a threedimensional structure-based method. Nat Microbiol 4: 112-123.

San Millan A, Pena-Miller R, Toll-Riera M, Halbert ZV, McLean AR, Cooper BS \& MacLean RC (2014) Positive selection and compensatory adaptation interact to stabilize non-transmissible plasmids. Nat Commun 5: 5208. 
Saylor C, Dadachova E \& Casadevall A (2009) Monoclonal antibody-based therapies for microbial diseases. Vaccine 27 Suppl 6: G38-46.

Schwarz S \& Johnson AP (2016) Transferable resistance to colistin: a new but old threat. $J$ Antimicrob Chemother 71: 2066-2070.

Segata N (2015) Gut Microbiome: Westernization and the Disappearance of Intestinal Diversity. Curr Biol 25: R611-613.

Segata N, Waldron L, Ballarini A, Narasimhan V, Jousson O \& Huttenhower C (2012) Metagenomic microbial community profiling using unique clade-specific marker genes. Nat Methods 9: 811-814.

Silva RF, Mendonça SCM, Carvalho LM, Reis AM, Gordo I, Trindade S \& Dionisio F (2011) Pervasive Sign Epistasis between Conjugative Plasmids and Drug-Resistance Chromosomal Mutations. PLoS Genet 7: e1002181.

Smillie CS, Smith MB, Friedman J, Cordero OX, David LA \& Alm EJ (2011) Ecology drives a global network of gene exchange connecting the human microbiome. Nature 480: 241-244.

Smith PA \& Romesberg FE (2007) Combating bacteria and drug resistance by inhibiting mechanisms of persistence and adaptation. Nat Chem Biol 3: 549-556.

Socha RD, Chen J \& Tokuriki N (2019) The Molecular Mechanisms Underlying Hidden Phenotypic Variation among Metallo-beta-Lactamases. J Mol Biol 431: 1172-1185.

Sommer MOA, Dantas G \& Church GM (2009) Functional characterization of the antibiotic resistance reservoir in the human microflora. Science 325: 1128-1131.

Sommer MOA, Munck C, Toft-Kehler RV \& Andersson DI (2017) Prediction of antibiotic resistance: time for a new preclinical paradigm? Nat Rev Microbiol 15: 689-696.

Sorg RA, Lin L, van Doorn GS, Sorg M, Olson J, Nizet V \& Veening JW (2016) Collective Resistance in Microbial Communities by Intracellular Antibiotic Deactivation. PLoS Biol 14: e2000631.

Stegger M, Wirth T, Andersen PS, et al. (2014) Origin and evolution of European communityacquired methicillin-resistant Staphylococcus aureus. MBio 5: e01044-01014.

Stevens DL, Gibbons AE, Bergstrom R \& Winn V (1988) The Eagle effect revisited: efficacy of clindamycin, erythromycin, and penicillin in the treatment of streptococcal myositis. J Infect Dis 158: $23-28$.

Stone LK, Baym M, Lieberman TD, Chait R, Clardy J \& Kishony R (2016) Compounds that select against the tetracycline-resistance efflux pump. Nat Chem Biol 12: 902.

Suez J, Zmora N, Zilberman-Schapira G, et al. (2018) Post-Antibiotic Gut Mucosal Microbiome Reconstitution Is Impaired by Probiotics and Improved by Autologous FMT. Cell 174: 1406-1423 e1416.

Thulin E, Thulin M \& Andersson DI (2017) Reversion of High-level Mecillinam Resistance to Susceptibility in Escherichia coli During Growth in Urine. EBioMedicine 23: 111-118.

Trindade S, Sousa A, Xavier KB, Dionisio F, Ferreira MG \& Gordo I (2009) Positive epistasis drives the acquisition of multidrug resistance. PLoS Genet 5: e1000578.

Tyers M \& Wright GD (2019) Drug combinations: a strategy to extend the life of antibiotics in the 21st century. Nat Rev Microbiol 17: 141-155.

Van Boeckel TP, Pires J, Silvester R, et al. (2019) Global trends in antimicrobial resistance in animals in low- and middle-income countries. Science $\mathbf{3 6 5}$.

van de Sande-Bruinsma N, Grundmann H, Verloo D, Tiemersma E, Monen J, Goossens H \& Ferech M (2008) Antimicrobial drug use and resistance in Europe. Emerg Infect Dis 14: 17221730. 
Wahid R, Fresnay S, Levine MM \& Sztein MB (2015) Immunization with Ty21a live oral typhoid vaccine elicits crossreactive multifunctional CD8+ T-cell responses against Salmonella enterica serovar Typhi, S. Paratyphi A, and S. Paratyphi B in humans. Mucosal Immunol 8: 1349-1359.

Wahid R, Fresnay S, Levine MM \& Sztein MB (2016) Cross-reactive multifunctional CD4+ T cell responses against Salmonella enterica serovars Typhi, Paratyphi A and Paratyphi B in humans following immunization with live oral typhoid vaccine Ty21a. Clin Immunol 173: 87-95.

Wales AD \& Davies RH (2015) Co-Selection of Resistance to Antibiotics, Biocides and Heavy Metals, and Its Relevance to Foodborne Pathogens. Antibiotics (Basel) 4: 567-604.

Walvekar P, Gannimani R \& Govender T (2019) Combination drug therapy via nanocarriers against infectious diseases. Eur J Pharm Sci 127: 121-141.

Wang R, van Dorp L, Shaw LP, et al. (2018) The global distribution and spread of the mobilized colistin resistance gene mcr-1. Nat Commun 9: 1179.

Webber MA, Whitehead RN, Mount M, Loman NJ, Pallen MJ \& Piddock LJ (2015) Parallel evolutionary pathways to antibiotic resistance selected by biocide exposure. J Antimicrob Chemother 70: 2241-2248.

Weigel LM, Clewell DB, Gill SR, et al. (2003) Genetic analysis of a high-level vancomycinresistant isolate of Staphylococcus aureus. Science 302: 1569-1571.

Weinroth MD, Scott HM, Norby B, et al. (2018) Effects of Ceftiofur and Chlortetracycline on the Resistomes of Feedlot Cattle. Appl Environ Microbiol 84.

Wistrand-Yuen E, Knopp M, Hjort K, Koskiniemi S, Berg OG \& Andersson DI (2018) Evolution of high-level resistance during low-level antibiotic exposure. Nat Commun 9: 1599.

Wright GD (2016) Antibiotic Adjuvants: Rescuing Antibiotics from Resistance. Trends Microbiol 24: 862-871.

Wu ML, Tan J \& Dick T (2015) Eagle Effect in Nonreplicating Persister Mycobacteria. Antimicrob Agents Chemother 59: 7786-7789.

Wyres KL \& Holt KE (2016) Klebsiella pneumoniae Population Genomics and AntimicrobialResistant Clones. Trends Microbiol 24: 944-956.

Yair Y \& Gophna U (2018) Pandemic Bacteremic Escherichia Coli Strains: Evolution and Emergence of Drug-Resistant Pathogens. Curr Top Microbiol Immunol 416: 163-180.

Yoon EJ, Grillot-Courvalin C \& Courvalin P (2017) New aminoglycoside-modifying enzymes $\mathrm{APH}\left(3^{\prime}\right)$-VIII and APH(3')-IX in Acinetobacter rudis and Acinetobacter gerneri. J Antibiot (Tokyo) 70: $400-403$.

Yoon EJ, Goussard S, Nemec A, Lambert T, Courvalin P \& Grillot-Courvalin C (2016) Origin in Acinetobacter gyllenbergii and dissemination of aminoglycoside-modifying enzyme AAC(6')-Ih. $J$ Antimicrob Chemother 71: 601-606.

Yu G, Baeder DY, Regoes RR \& Rolff J (2018) Predicting drug resistance evolution: insights from antimicrobial peptides and antibiotics. Proc Biol Sci 285.

Yurtsev EA, Conwill A \& Gore J (2016) Oscillatory dynamics in a bacterial cross-protection mutualism. Proc Natl Acad Sci U S A 113: 6236-6241.

Zheng G, Lu Y, Wang D \& Zhou L (2019) Importance of sludge conditioning in attenuating antibiotic resistance: Removal of antibiotic resistance genes by bioleaching and chemical conditioning with $\mathrm{Fe}[\mathrm{III}] / \mathrm{CaO}$. Water Res 152: 61-73.

Zhu L, Liu R, Liu T, Zou X, Xu Z \& Guan H (2019) A novel strategy to screen inhibitors of multiple aminoglycoside-modifying enzymes with ultra-high performance liquid chromatographyquadrupole-time-of-flight mass spectrometry. J Pharm Biomed Anal 164: 520-527. 
Zimmermann P \& Curtis N (2018) The effect of aspirin on antibiotic susceptibility. Expert Opin Ther Targets 22: 967-972. 